\title{
Reflectance fluctuations in an absorbing random waveguide
}

\author{
T. Sh. Misirpashaev \\ Instituut-Lorentz, University of Leiden, 2300 RA Leiden, the Netherlands; Landau \\ Institute for Theoretical Physics, 117334 Moscow, Russia \\ C. W. J. Beenakker \\ Instituut-Lorentz, University of Leiden, 2300 RA Leiden, the Netherlands
}

(Submitted 16 July 1996)

Pis'ma Zh. Éksp. Teor. Fiz. 64, No. 4, 289-293 (25 August 1996)

\begin{abstract}
We study the statistics of the reflectance (the ratio of reflected and incident intensities) of an $\mathrm{N}$-mode disordered waveguide with weak absorption $\gamma$ per mean free path. Two distinct regimes are identified. The regime $\gamma N^{2} \gg 1$ shows universal fluctuations. With increasing length $L$ of the waveguide, the variance of the reflectance changes from the value $2 / 15 N^{2}$, characteristic for universal conductance fluctuations in disordered wires, to another value $1 / 8 N^{2}$, characteristic for chaotic cavities. The weak-localization correction to the average reflectance performs a similar crossover from the value $1 / 3 N$ to $1 / 4 N$. In the regime $\gamma N^{2} \ll 1$, the large- $L$ distribution of the reflectance $R$ becomes very wide and asymmetric, $P(R) \propto(1-R)^{-2}$ for $R \ll 1-\gamma N$. (C) 1996 American Institute of Physics. [S0021-3640(96)01416-8]
\end{abstract}

PACS numbers: $05.40 .+\mathrm{j}$, 42.25.Bs, 78.20.Ci

An elegant and fundamental description of universal conductance fluctuations is provided by random-matrix theory. ${ }^{1}$ Different complex physical systems can be classified into a few universality classes, characterized by the dimensionality of the geometry and by the symmetries of the scattering matrix. The so-called circular ensemble, with a uniform distribution of the scattering matrix on the unitary group, describes chaotic cavities. ${ }^{2}$ The variance of the conductance in this ensemble (in units of $2 e^{2} / h$ ) equals $1 / 8 \beta$, where $\beta=1(\beta=2)$ for systems with (without) time-reversal symmetry in the absence of spin-orbit interaction. ${ }^{3}$ This is the zero-dimensional limit, corresponding to a logarithmic repulsion of the transmission and reflection eigenvalues. A disordered wire belongs to a different universality class (one-dimensional limit), with a nonlogarithmic eigenvalue repulsion and a variance $2 / 15 \beta$. The change from $1 / 8$ to $2 / 15$ is due to a weakening of the repulsion between the smallest transmission eigenvalues. ${ }^{4}$

The optical analogue of universal conductance fluctuations is the appearance of sample-to-sample fluctuations in the intensity transmitted or reflected by a random medium. Universality in this case means that the transmitted or reflected intensity, divided by the incident intensity per transverse mode of the medium, fluctuates with a variance which is independent of the sample size or the degree of disorder. ${ }^{5}$ It is essential for this universality that the incident illumination be diffusive, which means that the incident intensity is equally distributed over the $N$ transverse modes of the medium. A new aspect of the optical case is the possibility of absorption or amplification of radiation. In Ref. 6 
it was shown that the distribution of reflection eigenvalues in the limit of an infinitely long waveguide with absorption or amplification is the Laguerre ensemble of randommatrix theory. The reflection eigenvalues $R_{n}, n=1,2, \ldots N$, are the eigenvalues of the matrix product $r r^{\dagger}$, where $r$ is the $N \times N$ reflection matrix of the waveguide. In Ref. 6 the fluctuations in the reflected intensity were computed for the case of plane-wave illumination. The purpose of the present paper is to consider the case of diffusive illumination, in order to make the connection with universal conductance fluctuations.

We consider the reflection of monochromatic radiation with wavenumber $k$ by a waveguide of length $L$ and width $W$. The reflectance $R$ is defined as the ratio of reflected and incident intensities. For diffusive illumination it is given by

$$
R=N^{-1} \sum_{n, m}\left|r_{n m}\right|^{2}=N^{-1} \sum_{n} R_{n}
$$

The $L$-dependence of the distribution of reflection eigenvalues is described by the Fokker-Planck equation ${ }^{6}$

$$
\begin{aligned}
l \frac{\partial}{\partial L} P\left(\left\{\lambda_{n}\right\}, L\right)= & \frac{2}{\beta N+2-\beta} \sum_{i=1}^{N} \frac{\partial}{\partial \lambda_{i}} \lambda_{i}\left(1+\lambda_{i}\right) \\
& \times\left[\frac{\partial P}{\partial \lambda_{i}}+\beta P \sum_{j \neq i} \frac{1}{\lambda_{j}-\lambda_{i}}+\gamma(\beta N+2-\beta) P\right],
\end{aligned}
$$

where we use the parametrization $R_{n}=\lambda_{n} /\left(1+\lambda_{n}\right), \lambda_{n} \in(0, \infty)$. The parameter $\gamma>0$ is the ratio of the mean free path $l$ to the absorption length $l_{a}$. Equation (2) is valid if $k l$ $\gg 1, k l_{a} \gg 1$, and $W \ll L$. Optical systems normally possess time-reversal symmetry ( $\beta$ $=1$, but in view of a recently observed magneto-optical effect, ${ }^{7}$ we also include the case of broken time-reversal symmetry $(\beta=2)$.

Relevant length scales are defined in terms of the transmitted intensity. ${ }^{8,9}$ The transmitted intensity decays linearly with $L$ for $L \ll \xi$ and exponentially for $L \gg \xi$, where the decay length $\xi=\left(1 / \xi_{a}+1 / \xi_{l}\right)^{-1}$ contains a contribution $\xi_{a}=l\left(2 \gamma+\gamma^{2}\right)^{-1 / 2}$ from absorption and a contribution $\xi_{l}=\frac{1}{2} l(\beta N+2-\beta)$ from localization by disorder. We will study the two regimes (1) $\xi_{a} \ll \xi_{l}$ (or $\gamma N^{2} \gg 1$ ) and (2) $\xi_{a} \gg \xi_{l}$ (or $\gamma N^{2} \ll 1$ ). In both regimes we assume $N \gg 1$.

(1) The regime $\gamma N^{2} \gg 1$ admits of a perturbative treatment for all $L$ by the method of moments of Mello and Stone. ${ }^{10}$ We define the moments $M_{q}=N^{-1}(-1)^{q} \Sigma_{i}(1$ $\left.+\lambda_{i}\right)^{-q}$, so that $R=1+M_{1}$. The Fokker-Planck equation (2) enables us to express derivatives $\partial\left\langle M_{q_{1}}^{n_{1}} \ldots M_{q_{k}}^{n_{k}}\right\rangle / \partial L$ in terms of the moments themselves. Expanding each moment in powers of $1 / N$, we get a closed system of first-order differential equations for the coefficients. The method has been explained in detail, ${ }^{10}$ therefore we just present the result. We need the following terms in the $1 / N$-expansion of the moments,

$$
\begin{aligned}
& \left\langle M_{1}^{q}\right\rangle=F_{q, 0}+N^{-1} F_{q, 1}+N^{-2} F_{q, 2}+\mathscr{Q}\left(N^{-3}\right), \\
& \left\langle M_{1}^{q} M_{2}\right\rangle=G_{q, 0}+N^{-1} G_{q, 1}+\mathscr{O}\left(N^{-2}\right),
\end{aligned}
$$




$$
\left\langle M_{1}^{q} M_{3}\right\rangle=H_{q}+\mathscr{Q}\left(N^{-1}\right) .
$$

The $q$-dependence of the coefficients is given by

$$
\begin{aligned}
& F_{q, 0}=f^{q}, \quad F_{q, 1}=\delta_{\beta 1} q \mu f^{q-1}, \quad F_{q, 2}=q \eta_{\beta} f^{q-1}+q(q-1) \theta_{\beta} f^{q-2}, \\
& G_{q, 0}=g f^{q}, \quad G_{q, 1}=\delta_{\beta 1}\left(\sigma f^{q}+q \mu g f^{q-1}\right), \\
& H_{q}=h f^{q} .
\end{aligned}
$$

Here $f, g, h, \mu, \theta_{1,2}, \sigma, \eta_{1,2}$ are functions of $L$ which obey the following system of first-order differential equations and initial conditions:

$$
\begin{aligned}
& l d f / d L=f^{2}-2 \gamma f-2 \gamma, \quad f(0)=-1, \\
& l d g / d L=4(f-\gamma) g+2\left(f^{2}-2 \gamma f\right), \quad g(0)=1, \\
& l d h / d L=6(f-\gamma) h+3 g(g+2 f-2 \gamma), \quad h(0)=-1, \\
& l d \mu / d L=2(f-\gamma) \mu+g-f^{2}, \quad \mu(0)=0, \\
& l d \theta_{\beta} / d L=4(f-\gamma) \theta_{\beta}+(2 / \beta)(g+h)+\delta_{\beta 1} \mu\left(g-f^{2}\right), \quad \theta_{\beta}(0)=0, \\
& l d \sigma / d L=4(f-\gamma) \sigma+4 \mu(g+f-\gamma)+2\left(g-f^{2}\right)+4(h-f g), \quad \sigma(0)=0, \\
& l d \eta_{\beta} / d L=2(f-\gamma) \eta_{\beta}+2 \theta_{\beta}+\delta_{\beta 1}\left(\sigma-2 \mu f-g+f^{2}\right), \quad \eta_{\beta}(0)=0 .
\end{aligned}
$$

The equations can be easily solved one after another. The first two of them determine the mean and variance of the reflectance for plane-wave illumination, studied in Ref. 6 . The other equations determine the mean and the root-mean-squared fluctuations for diffusive illumination to order $N^{-1}$. We decompose $\langle R\rangle=R_{0}+\delta R$, where $R_{0}=\mathscr{Q}\left(N^{0}\right)$ and $\delta R$ $=\mathscr{Q}\left(N^{-1}\right)$. In terms of the functions in Eq. (5) we have

$$
\begin{aligned}
& R_{0}=1+f, \quad \delta R=\delta_{\beta 1} \mu / N, \\
& \operatorname{var} R=\left(2 \theta_{\beta}-\delta_{\beta 1} \mu^{2}\right) / N^{2}=4 \theta_{2} / \beta N^{2} .
\end{aligned}
$$

The weak-localization correction $\delta R$ vanishes for $\beta=2$, while var $R \propto 1 / \beta$, just as without absorption.

To find $\langle R\rangle$ and var $R$ we need to solve the first five equations (5). The analytical solution is cumbersome to use, but it is easy to integrate the system numerically at a given $\gamma$. Results for $\gamma=10^{-4}$ are shown in Fig. 1 . The large- $L$ limit can be found directly by replacing the derivatives at the left-hand side of Eq. (5) by zero. In this way, we obtain the following asymptotical values:

$$
\begin{aligned}
& R_{0}=1+\gamma-\sqrt{2 \gamma+\gamma^{2}}, \\
& \delta R=\delta_{\beta 1} N^{-1}\left(\frac{1}{2}(2+\gamma)^{-1}+\gamma-(1+\gamma) \sqrt{\gamma(2+\gamma)^{-1}}\right), \\
& \operatorname{var} R=\left[2 \beta N^{2}(2+\gamma)^{2}\right]^{-1} .
\end{aligned}
$$

The large- $L$ limit (7) an also be obtained from the Laguerre ensemble for the reflection eigenvalues, ${ }^{6}$ 


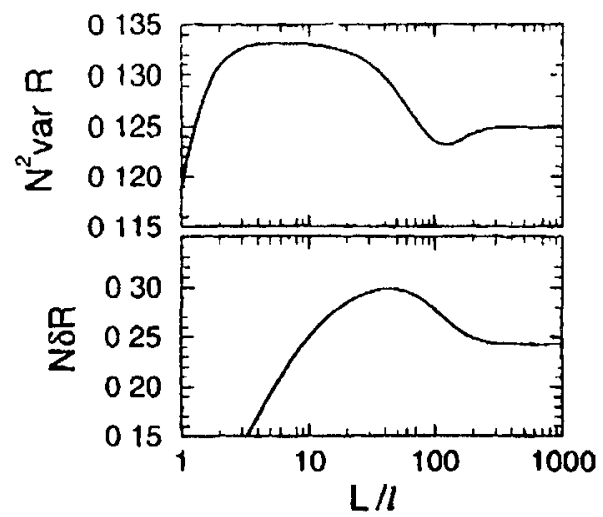

FIG 1 Length dependence of $N \delta R$ and $N^{2}$ var $R$ for $\beta=1$ and $\gamma=10^{-4}$, according to Eqs (5) and (6), un the regime $\gamma N^{2} \gg 1$ The vanance var $R$ of the reflectance crosses over from a plateau at $2 / 15 N^{2}$ (one dimensional limit) to a plateau at $1 / 8 N^{2}$ (zero dimensional limit) The crossover is nonmonotonic and occurs when the length of the waveguide becomes comparable to the decay length $\xi=70 l$ of the transmittance The weak-localization correction $\delta R$ shows a similar crossover from $1 / 3 N$ to $1 / 4 N$, but the plateaus are less well defined for this value of $\gamma$

$$
P_{\infty}\left(\left\{\lambda_{n}\right\}\right) \propto \exp \left[\beta \sum_{l<J} \ln \left|\lambda_{J}-\lambda_{t}\right|-\gamma(\beta N+2-\beta) \sum_{l} \lambda_{l}\right],
$$

which is the asymptotic solution of the Fokker-Planck equation (2) The limiting values (7) are reached when $L \gg \xi$, hence when the transmittance through the waveguide has become exponentially small. For $L \ll \xi$ the effect of absorption can be neglected. Over a range of lengths $L$ such that $l \ll L \ll \xi$, the mean and variance are given by ${ }^{10} R_{0}=1$ $-l / L, \delta R=\delta_{\beta 1} / 3 N$, var $R=2 / 15 \beta N^{2}$. The value $1 / 8 \beta N^{2}$ for the variance when $\gamma \ll 1$ and $L \gg \xi$ follows directly from the logarithmic repulsion of the $\lambda_{n}$ 's in the Laguerre ensemble (Ref. 11) ${ }^{\text {a) }}$ The difference with the $2 / 15 \beta N^{2}$ for $l \ll L \ll \xi$ arises because the repulsion is nonlogarithmic in the absence of absorption. ${ }^{4}$

(2) We now turn to the second regime, $\gamma N^{2} \ll 1$. This regime cannot be treated perturbatively for $L>\xi$, because of the onset of localization by disorder. The limiting large- $L$ values of $\langle R\rangle$ and var $R$ can be computed from Eq. (8) using formulas ${ }^{12}$ for the density and correlation function of the $\lambda_{n}$ 's in the Laguerre ensemble. The result is

$$
\begin{aligned}
& \langle R\rangle=1-\beta \gamma N\left[\ln \left(1 / \gamma N^{2}\right)+\mathscr{O}(1)\right], \\
& \operatorname{var} R=\beta \gamma-(\beta \gamma N)^{2}\left[\ln \left(1 / \gamma N^{2}\right)+\bigcirc(1)\right]^{2}
\end{aligned}
$$

A new crossover length $L_{c}=\xi \ln \left(1 / \gamma N^{2}\right)$ appears at which the mean and variance of $R$ attain their asymptotic values (9) This is the length at which the exponential decay $\langle 1-R\rangle \approx N^{-1} \exp (-L / 4 \xi)^{13}$ comes to a halt. 


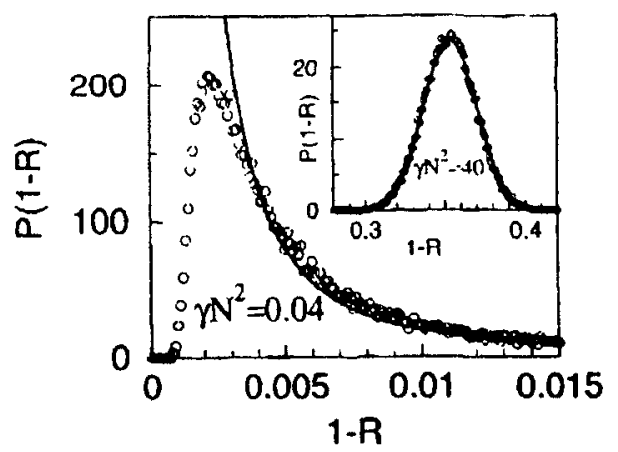

FIG. 2. Large- $L$ distribution of the reflectance for $\beta=1$. Data points are obtained by generating $5 \cdot 10^{4}$ random matrices in the Laguerre ensemble (8). The main plot is for the regime $\gamma N^{2} \ll 1\left(N=20, \gamma=10^{-4}\right)$, the solid curve being the asymptotic tail (10). The inset is for the regime $\gamma N^{2} \gg 1(N=20, \gamma=0.1)$, the solid curve being a Gaussian with mean and variance given by Eq. (7).

From Eq. (9) we see that the reflectance has a wide distribution in the regime $\gamma N^{2} \ll 1$ : The root-mean-squared fluctuations of $1-R$ are greater than the mean by a factor $\left(\gamma N^{2}\right)^{-1 / 2}$. (In the opposite regime $\gamma N^{2} \gg 1$, in contrast, the distribution of the reflectance is a narrow Gaussian, see the inset in Fig. 2.) To determine the tail of the distribution, it is sufficient to consider only the contribution from the smallest eigenvalue $\lambda_{1}$, which gives the main contribution to $1-R$ when $\gamma N^{2} \rightarrow 0$. The smallest eigenvalue in the Laguerre ensemble has the exponential distribution $P\left(\lambda_{1}\right)=\beta \gamma N^{2}$ exp $\left(-\beta \gamma N^{2} \lambda_{1}\right)$

$$
P(R)=\beta \gamma N(1-R)^{-2}, \quad \gamma N \ll 1-R<1 / N .
$$

We have calculated $P(R)$ by generating a large number of random matrices in the Laguerre ensemble (see Fig. 2). The distribution reaches its maximum at $1-R \simeq \gamma N$ and then drops to zero for smaller values of $1-R$. The tail for large values of $1-R$ is well described by Eq. (10) (solid curve in Fig. 2).

To conclude, we have studied the statistics of the reflectance in an absorbing random waveguide under diffusive illumination. When the decay of the transmittance is dominated by absorption $\left(\gamma N^{2} \gg 1\right)$, the fluctuations are shown to possess the same features as universal conductance fluctuations, including independence on the disorder and $1 / \beta$ dependence on the symmetry index. A crossover from the zero-dimensional to the onedimensional limit was found in Refs. 15 and 16 for a chain of chaotic cavities. (A long chain of cavities behaves as a diffusive wire.) We have found an opposite crossover, from the one-dimensional to the zero-dimensional limit, as the length of the waveguide is increased beyond the decay length $\xi$. Another regime, where the decay of the transmittance is dominated by localization due to disorder $\left(\gamma N^{2} \ll 1\right)$, is principally new and characterized by a wide and asymmetric distribution of the reflectance. The asymptotic regime is established at a new characteristic scale $L_{c}>\xi$. 
We thank P. W. Brouwer for helpful discussions. This work was supported by the Nederlandse Organisatie voor Wetenschappelijk Onderzoek (NWO) and the Stichting voor Fundamenteel Onderzoek der Materie (FOM).

a) The deviation of var $R$ from its universal value $1 / 8 \beta$ when $\gamma$ is not $\ll 1$ arises because the spectrum of the $\lambda_{n}$ 's has an upper bound at $\lambda=2 / \gamma$ (see C. W. J. Beenakker, Nucl. Phys. B 422, 515 (1994)).

\footnotetext{
${ }^{1}$ A. D. Stone, P. A. Mello, K. A. Muttalib, and J.-L. Pichard, in Mesoscopic Phenomena in Solids, Eds. B. L. Al'tshuler, P. A. Lee, and R. A. Webb, North Holland, Amsterdam, 1991; C. W. J. Beenakker, Rev. Mod. Phys., to appear.

${ }^{2}$ R. Blümel and U. Smilansky, Phys. Rev. Lett. 64, 241 (1989).

${ }^{3}$ H. U. Baranger and P. A. Mello, Phys. Rev. Lett. 73, 142 (1994); R. A. Jalabert, J.-L. Pichard, and C. W. J. Beenakker, Europhys. Lett. 27, 255 (1994).

${ }^{4}$ C. W. J. Beenakker and B. Rejaei, Phys. Rev. Lett. 71, 3689 (1993).

${ }^{5}$ P. A. Mello, E. Akkermans, and B. Shapiro, Phys. Rev. Lett. 61, 459 (1988); M. J. Stephen, in Mesoscopic Phenomena in Solids, Eds. B. L. Al'tshuler, P. A. Lee, and R. A. Webb, North Holland, Amsterdam, 1991.

${ }^{6}$ C. W. J. Beenakker, J. C. J. Paasschens, and P. W. Brouwer, Phys. Rev. Lett. 76, 1368 (1996).

${ }^{7}$ G. L. J. A. Rikken and B. A. van Tiggelen, Nature 381, 54 (1996).

${ }^{8}$ O. N. Dorokhov, Zh. Éksp. Teor. Fiz. 85, 1040 (1983) [Sov. Phys. JETP 58, 606 (1983)].

${ }^{9}$ T. Sh. Misirpashaev, J. C. J. Paasschens, and C. W. J. Beenakker, unpublished.

${ }^{10}$ P. A. Mello, Phys. Rev. Lett. 60, 1089 (1988); P. A. Mello and A. D. Stone, Phys. Rev. B 44, 3559 (1991).

${ }^{11}$ C. W. J. Beenakker, Phys. Rev. B 47, 15763 (1993).

${ }^{12}$ T. Nagao and K. Slevin, J. Math. Phys. 34, 2075, 2317 (1993).

${ }^{13}$ M. R. Zirnbauer, Phys. Rev. Lett. 69, 1584 (1992).

${ }^{14}$ A. Edelman, Linear Algebra Appl. 159, 55 (1991); P. J. Forrester, Nucl. Phys. B 402, 709 (1993).

${ }^{15}$ S. lida, H. A. Weidenmüller, and J. A. Zuk, Phys. Rev. Lett. 64, 583 (1990).

${ }^{16}$ N. Argaman, Phys. Rev. B 53, 7035 (1996).
}

Published in English in the original Russian journal. Edited by J. R. Anderson. 